\title{
Cardiovascular risk and inflammation in rheumatic diseases
}

\author{
Armen Yuri Gasparyan ${ }^{1}$
}

Published online: 24 November 2016

(C) Springer-Verlag Berlin Heidelberg 2016

Cardiovascular manifestations of rheumatic diseases have been thoroughly examined over the past decades. It has been established that all structures of the heart can be affected due to the long-term systemic inflammation and specific autoimmune and autoinflammatory factors [1]. Classic clinical models of cardiovascular phenomenon in rheumatic diseases are rheumatoid arthritis (RA) and systemic lupus erythematosus (SLE), which are associated with enhanced course of atherosclerosis and various atherothrombotic events. Numerous large cohort studies of RA and SLE have provided evidence of atypical and severe course of myocardial infarction and heart failure in these autoimmune diseases, necessitating specific early diagnostic and preventive approaches.

The intensity of systemic inflammation confounds cardiovascular manifestations across rheumatic diseases. Accordingly, inflammation-induced atherosclerosis is believed to be a more pressing issue in RA and SLE than in autoinflammatory disorders [2]. Methotrexate therapy improves lipid profiles and reduces atherothrombotic risk in RA by diminishing production of $\mathrm{C}$-reactive protein (CRP) and suppressing systemic inflammation [3]. Biologic agents may also protect from enhanced atherogenesis in RA by potently suppressing pro-inflammatory cytokines, such as interleukin-6 and tumor necrosis factor alpha [4]. Although there are no specifically designed trials of colchicine therapy in atherogenesis due autoinflammatory

Armen Yuri Gasparyan

a.gasparyan@gmail.com

1 Departments of Rheumatology and Research and Development, Dudley Group NHS Foundation Trust (Teaching Trust of the University of Birmingham, UK), Russells Hall Hospital, Dudley, West Midlands DY1 2HQ, UK disorders, this anti-neutrophilic agent is viewed as cardioprotective and essential for cohorts of patients with inflammatory amyloidosis and pericarditis [5].

Case series and pilot cohort studies of atherogenesis in vasculitides provide variable results, which may be related to the intensity of systemic inflammation, specific pathogenic factors, and differing anti-inflammatory therapies, some of which, and particularly glucocorticosteroids, may adversely affect lipid profiles, glucose and insulin levels, platelet function, and other thrombotic factors. Behcet's disease (BD) was shown to present with intracardiac thromboses and coronary aneurysms, but not with enhanced atherosclerosis [6]. Likewise, macrovascular atherosclerotic disease was not enhanced in giant cell arteritis [7]. Nonetheless, when compared with SLE, patients with Takayasu arteritis (TA) had more atherosclerotic plaques despite comparable cardiovascular risk factors [8].

There have been several attempts to propose markers for early diagnosis and monitoring of cardiovascular disease in rheumatic disorders. Most related studies have focused on RA. One of the well-designed prospective cohort studies with 138 RA patients, who were followed from 2007 to 2013, proved that carotid plaques, intima-media thickness, and aortic pulse wave velocity predict cardiovascular events [9]. The role of many other vascular and cellular markers for predicting the course of cardiovascular disease and heart failure in inflammatory rheumatic diseases still remains poorly explored and not validated. The issue is further complicated with uncertainties associated with predictive value of vascular markers in case of combination of rheumatic diseases, other comorbidities, and across various age and ethnic cohorts. Vascular marker studies are predominantly conducted in the USA and Western Europe and are hardly applicable in the rest of the world. 
European recommendations of cardiovascular risk assessment and management in rheumatic diseases are currently available and are largely based on cohort studies of RA. These recommendations may be applicable to ankylosing spondyloarthritis (AS) and psoriatic arthritis (PsA), though related evidence-base is still not as strong as in RA. The most important point for rheumatologists managing inflammatory joint diseases is that suppressing systemic inflammation and controlling disease activity are beneficial for reducing related cardiovascular risk. Regularly assessing lipid profile and screening for carotid atherosclerotic plaques are advisable. The obtained results may guide lifestyle modifications and therapies with use of statins and antihypertensive drugs. The recommendations emphasized that the use of non-steroidal anti-inflammatory drugs (NSAIDs) and corticosteroids should be justified in view of the presence of cardiovascular risk factors and established cardiovascular disease [10].

Despite several attempts to quantify cardiovascular risk in inflammatory joint diseases, there are still no globally acceptable recommendations. The least rheumatologists can do at this stage without use of risk scores is to properly manage the course of systemic inflammation, avoid side effects of anti-inflammatory drugs, monitor vascular function and extent of atherosclerosis, and advise lifestyle modifications. The latter may include, but not limited to smoking cessation, regular physical activity, healthy diet, all of which bring additive anti-inflammatory and antithrombotic benefits.

With the above mentioned in mind, readers of this issue are invited to reflect on carefully selected original research papers, reviews, and case studies and propose new directions for research on cardiovascular issues in rheumatic diseases.

\section{References}

1. Prasad M, Hermann J, Gabriel SE, Weyand CM, Mulvagh S, Mankad R, Oh JK, Matteson EL, Lerman A (2015) Cardiorheumatology: cardiac involvement in systemic rheumatic disease. Nat Rev Cardiol 12(3):168-176

2. Gasparyan AY, Stavropoulos-Kalinoglou A, Mikhailidis DP, Toms TE, Douglas KM, Kitas GD (2010) The rationale for comparative studies of accelerated atherosclerosis in rheumatic diseases. Curr Vasc Pharmacol 8(4):437-449

3. Popkova TV, Novikova DS, Gasparyan AY, Nasonov EL (2015) Cardiovascular effects of methotrexate in rheumatoid arthritis revisited. Curr Med Chem 22(16):1903-1910

4. Chen DY, Chen YM, Hsieh TY, Hsieh CW, Lin CC, Lan JL (2015) Significant effects of biologic therapy on lipid profiles and insulin resistance in patients with rheumatoid arthritis. Arthritis Res Ther 17:52

5. Gasparyan AY, Ayvazyan L, Yessirkepov M, Kitas GD (2015) Colchicine as an anti-inflammatory and cardioprotective agent. Expert Opin Drug Metab Toxicol 11(11):1781-1794

6. Seyahi E, Ugurlu S, Cumali R, Balci H, Ozdemir O, Melikoglu M, Hatemi G, Fresko I, Hamuryudan V, Yurdakul S, Yazici $\mathrm{H}$ (2008) Atherosclerosis in Behçet's syndrome. Semin Arthritis Rheum 38(1):1-12

7. Gonzalez-Juanatey C, Lopez-Diaz MJ, Martin J, Llorca J, Gonzalez-Gay MA (2007) Atherosclerosis in patients with biopsyproven giant cell arteritis. Arthritis Rheum 57(8):1481-1486

8. Seyahi E, Ugurlu S, Cumali R, Balci H, Seyahi N, Yurdakul S, Yazici H (2006) Atherosclerosis in Takayasu arteritis. Ann Rheum Dis 65(9):1202-1207

9. Ikdahl E, Rollefstad S, Wibetoe G, Olsen IC, Berg IJ, Hisdal J, Uhlig T, Haugeberg G, Kvien TK, Provan SA, Semb AG (2016) Predictive value of arterial stiffness and subclinical carotid atherosclerosis for cardiovascular disease in patients with rheumatoid arthritis. J Rheumatol 43(9):1622-1630

10. Agca R, Heslinga SC, Rollefstad S, Heslinga M, McInnes IB, Peters MJ et al (2016) EULAR recommendations for cardiovascular disease risk management in patients with rheumatoid arthritis and other forms of inflammatory joint disorders: 2015/2016 update. Ann Rheum Dis. doi:10.1136/annrheumdis-2016-209775 (Epub ahead of print) 